\title{
Spectral refractive index assessment of turbid samples by combining spatial frequency near-infrared spectroscopy with Kramers-Kronig analysis
}

\author{
Omri Meitav \\ Oren Shaul \\ David Abookasis
}




\title{
Spectral refractive index assessment of turbid samples by combining spatial frequency near-infrared spectroscopy with Kramers-Kronig analysis
}

\author{
Omri Meitav, Oren Shaul, and David Abookasis* \\ Ariel University, Department of Electrical and Electronics Engineering, Ariel, Israel
}

\begin{abstract}
A practical algorithm for estimating the wavelength-dependent refractive index (RI) of a turbid sample in the spatial frequency domain with the aid of Kramers-Kronig (KK) relations is presented. In it, phase-shifted sinusoidal patterns (structured illumination) are serially projected at a high spatial frequency onto the sample surface (mouse scalp) at different near-infrared wavelengths while a camera mounted normally to the sample surface captures the reflected diffuse light. In the offline analysis pipeline, recorded images at each wavelength are converted to spatial absorption maps by logarithmic function, and once the absorption coefficient information is obtained, the imaginary part ( $k$ ) of the complex RI (CRI), based on Maxell's equations, can be calculated. Using the data represented by $k$, the real part of the CRI $(n)$ is then resolved by KK analysis. The wavelength dependence of $n(\lambda)$ is then fitted separately using four standard dispersion models: Cornu, Cauchy, Conrady, and Sellmeier. In addition, three-dimensional surface-profile distribution of $n$ is provided based on phase profilometry principles and a phase-unwrapping-based phase-derivative-variance algorithm. Experimental results demonstrate the capability of the proposed idea for sample's determination of a biological sample's RI value. @ 2018 Society of Photo-Optical Instrumentation Engineers (SPIE) [DOI: 10.1117/1.JBO.23.3.035007]
\end{abstract}

Keywords: refractive index; spatial frequency; Kramers-Kronig relations; dispersion models; surface profile.

Paper 180016R received Jan. 10, 2018; accepted for publication Mar. 9, 2018; published online Mar. 28, 2018.

\section{Introduction}

The complex refractive index (CRI), which reflects a sample's intrinsic properties, is composed of real $(n)$ and imaginary $(k)$ parts as: $\mathrm{CRI}=n+i k . n$ and $k$ are the positive real functions reflecting the phase velocity $(C / V)$ of light waves propagated and its attenuation, respectively. ${ }^{1-3}$ In addition to that, CRI is frequently utilized to solve boundary questions at the interferences when modeling light migration within heterogeneous samples, such as biological tissues. ${ }^{4}$ In the context of biomedical optics, $n$ is related to the scattering coefficient $\left(\mu_{\mathrm{s}}\right),{ }^{5}$ whereas the imaginary part $(k)$ is connected to absorption coefficient $\left(\mu_{\mathrm{a}}\right)$ as given by: $k=\lambda \mu_{\mathrm{a}} / 4 \pi$. $^{6,7}$ Therefore, exploiting the changes in CRI parameters can provide useful information regarding the sample's physiological properties during diagnostic or treatment procedures. Overall, the independent derivation of $n$ and $k$ values, as well as the determination of scattering and absorption as functions of wavelength, can provide a comprehensive battery of biochemical, morphological, and histochemical parameters of biological tissue. Along this line, once the spectral data of $k$ are obtained, $n$ can be deduced via Kramers-Kronig (KK) relations and vice versa. The KK relations, dispersion relations wellknown in the context of optical spectroscopy, connect between these variables as follows: ${ }^{8}$

$n(\lambda) \approx n_{o}+\frac{2}{\pi} P \int_{\lambda_{1}}^{\lambda_{n}} \frac{k\left(\lambda^{\prime}\right) \lambda^{\prime}}{\lambda^{2}-\left(\lambda^{\prime}\right)^{2}} \mathrm{~d} \lambda^{\prime}$,

where $\lambda$ is the wavelength of radiation, $P$ denotes the Cauchy principle value, and $n_{o}$ is the high-wavelength refractive index (RI), ${ }^{9}$ approximately $n_{o} \approx 1.38$ at $650 \mathrm{~nm}$ for biological tissue. ${ }^{10}$
Over the past decades, several approaches have been developed to determine the RI of dense random media, such as biological tissue, each method possessing its advantages and disadvantages. In this work, we suggest the application of spatially modulated near-infrared (NIR) illumination [also known as modulated imaging (MI)] to derive sample RI. MI is a recently developed, noncontact, scan-free, and wide-field imaging approach, which is well-suited for quantitative spatial mapping of optical properties and biochemical composition (such as hemoglobin, lipid, water, etc.) of highly scattering media, such as biological tissue. ${ }^{11}$ The key advantage of this platform stems from its use of structured illumination (i.e., stripes of light) in at least two spatial frequencies and phase offsets to decouple light scattering upon absorption and performing sample optical sectioning. In general, lower spatial frequency patterns are primarily sensitive to absorption, whereas scattering strongly dominates the higher frequencies. ${ }^{12-14}$ In turn, tissue absorption is typically linked to metabolic properties, such as perfusion, oxygenation, and chemical content, ${ }^{15}$ whereas tissue scattering is more related to structural properties, such as cell density or tissue type. ${ }^{16}$ In our setup, sinusoidal light patterns at three different offset phases ( $0 \mathrm{deg}, 120 \mathrm{deg}$, and $240 \mathrm{deg}$ ) at a single high spatial frequency $\left(0.27 \mathrm{~mm}^{-1}\right)$ are serially projected onto the sample surface at six discrete NIR wavelengths. Next, the diffusely reflected light (spatial-modulated reflected images) is captured by a CCD camera fixed normally to the sample surface and stored for offline analysis wherein recorded images at each excitation wavelength $\left(\lambda_{i}, i=1\right.$ to 6$)$ are processed and converted to spatial absorption coefficient maps $\left[\mu_{\mathrm{a}}\left(x, y, \lambda_{i}\right)\right]$. Once the values of $\mu_{\mathrm{a}}\left(x, y, \lambda_{i}\right)$ are obtained, the discrete $k\left(\lambda_{i}\right)$ 
data are calculated $\left[k\left(\lambda_{i}\right),=\lambda_{i} \mu_{\mathrm{a}}\left(\lambda_{i}\right) / 4 \pi\right]^{6,7}$ and fitted by the power-law equation $\left[k(\lambda)=a \lambda^{1-b}\right]^{17}$ to cover a wide wavelength spectrum, increasing the accuracy of the calculated $n(\lambda)$. Finally, the wavelength dependence of $n(\lambda)$ is fitted according to a standard dispersion model. ${ }^{18,19}$ The steps leading to RI reconstruction are illustrated schematically in Fig. 2 and are elaborated in Sec. 2. As stated above, while there are several methods to derive $n(\lambda)$, its derivation by spatially modulated illumination is more simple and easy, which grants additional advantages to this technique compared to the others.

Similarly, to the MI system, structured light (fringe) projections based phase-shifting [phase-measuring profolimetry (PMP)] approaches offer an optical metrological tool for threedimensional (3-D) reconstruction of object shape in many areas, including industrial inspection, medical engineering, machine vision, and aviation, due to its accuracy, speed, and nonrequirement of contact. ${ }^{20-22} 3-\mathrm{D}$ object reconstruction is achieved via absolute phase mapping retrieved by arctangent function calculations of the captured fringe patterns followed by an unwrapping algorithm to remove phase discontinuities and obtaining continued phase distribution. Since PMP and MI both utilize the same working principle, they may be implemented complimentarily. Thus, these two techniques may be utilized to create a quantitative 3-D RI map, without any specialized hardware or software. The complementary use of PMP and MI was recently described by van de Giessen et al. ${ }^{23}$ for 3-D profiling of sample absorption and scattering properties. Recently, the use of spatial frequency domain imaging combined with PMP to determine the optical properties of a sample was also demonstrated. ${ }^{24}$ In this study, we implement a different approach to achieve the same and to additionally calculate the RI of biological tissue.

\section{Materials and Methods}

\subsection{Instrumentation}

Figure 1 provides a schematic representation of the current optical setup, consisting of four main hardware components: a modified commercial digital light projector (DLP) projector

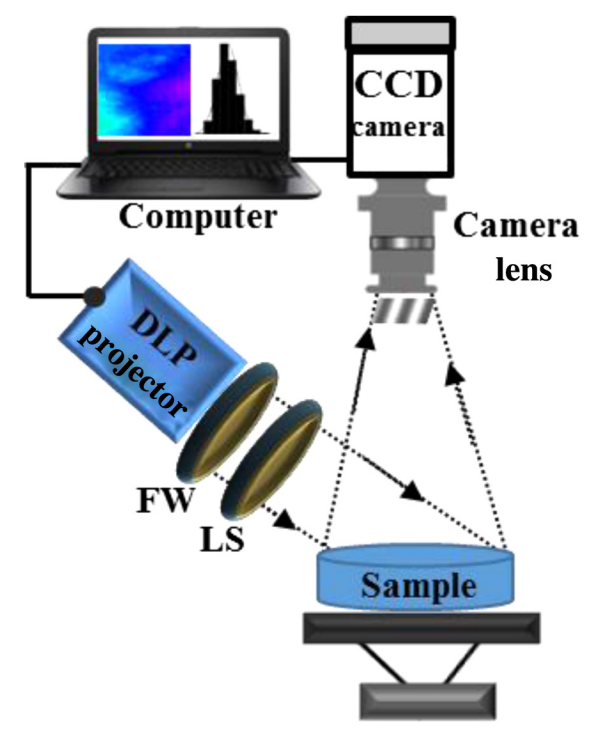

Fig. 1 Schematic diagram of the experimental system: DLP, digital light projector; FW, filter wheel; LS, lens system; and CCD, chargecoupled device.
(PLUS, U5-112), filter wheel (Thorlabs, FW102C), a 14-bit CCD camera $[656(\mathrm{~h}) \times 494(\mathrm{v})$ pixels; 121 fps; Guppy Pro, F-031B, AVT] equipped with a zoom imaging lens system (Computar) positioned above the sample, and a personal computer (Ipex T300). The filter wheel is equipped with six narrow bandpass filters centered at wavelengths of $690,780,800,880$, 920, and $970 \mathrm{~nm}$ (Thorlabs, FB Series, BW $\pm 10 \mathrm{~nm}$ ). At each excitation wavelength, sinusoidal patterns (sine function in MATLAB ${ }^{\mathrm{TM}}$ ) are sequentially projected by the projector onto the sample surface three times at single high spatial frequency of $0.27 \mathrm{~mm}^{-1}$ with phase offsets of $0 \mathrm{deg}, 120 \mathrm{deg}$, and $240 \mathrm{deg}$, respectively. We used these three demodulated images to determine the scattering and absorption coefficients and to create 3-D surface construction. The diffuse reflected (remitted reflectance) light that passes through the lens system is then captured by CCD camera and saved in TIFF format for offline processing and analysis (performed by Dell Inspiration 15, 3000 Series, Core $i 5)$. Camera gain and exposure time were adjusted for each illumination wavelength during the calibration process to enable high signal-to-noise ratio while avoiding pixel saturation. Each single repetition lasted up to $\sim 1.5 \mathrm{~min}$ and included a total of 18 captured images: one spatial frequency $\times 3$ phases $\times$ 6 wavelengths. More rapid imaging could be achieved using a camera with higher quantum efficiency at longer wavelengths. The entire setup is controlled by custom MATLAB ${ }^{\mathrm{TM}}$ software (Version R2013b, MathWorks Inc., Natick, Massachusetts) such that imaging acquisition, synchronization, and data processing were carried out using in-house developed MATLAB ${ }^{\mathrm{TM}}$ scripts.

\subsection{Imaging Procedure and Processing}

Throughout the experiments, raw reflected light image data of the sample were recorded by CCD camera while projecting spatially modulated NIR light onto the sample surface. Each image spanned a $10 \mathrm{~mm} \times 10 \mathrm{~mm}$ field of view (FOV) using the central 656 pixels $\times 494$ pixels of the sensor and was composed from 30 consecutive frames/second averaged into a single reflected image. Image sets from each of the six wavelengths were acquired at the high spatial frequency of $0.27 \mathrm{~mm}^{-1}$. Complete data sets were acquired every $\sim 1.5 \mathrm{~min}$; each repetition including 18 consecutive images: 3 phase shifts each at 6 wavelengths. Overall, 10 repetitions were performed for each wavelength and then were averaged to increase the image signal-to-noise ratio, which underwent preprocessing using the "fspecial" function in MATLAB" to improve image quality. A region-of-interest $(\mathrm{ROI})(150 \times 150$ pixels $)$ was selected using the "imfreehand" MATLABTM function. No other processing has been applied to the images. The absorption coefficient of the selected ROI image at wavelength, $\lambda_{i}$, was then derived by taking the negative logarithm of the uniform illumination as

$\mu_{\mathrm{a}}\left(\lambda_{\mathrm{i}}\right)=\mathrm{k}_{\mathrm{a}}\left(\lambda_{\mathrm{i}}\right) \times\left\{-\log _{10}\left[\mathrm{R}_{\mathrm{d}}^{\mathrm{DC}}\left(\lambda_{\mathrm{i}}\right)\right]\right\}$

where $R_{\mathrm{d}}^{\mathrm{DC}}\left(\lambda_{i}\right)$ represents the reflectance of uniform illumination $\left(f_{x}=0 \mathrm{~mm}^{-1}\right)$ obtained by averaging of three recorded spatially phased images: $I_{\mathrm{o}}\left(\lambda_{\mathrm{i}}\right), I_{120}\left(\lambda_{\mathrm{i}}\right)$, and $I_{240}\left(\lambda_{\mathrm{i}}\right)$. From the obtained $R_{\mathrm{d}}^{\mathrm{DC}}\left(\lambda_{i}\right)$ image, the mean and standard deviation ("mean2" and "std2" functions) values of the absorption are derived at each wavelength. 


\subsection{Calibration}

The calibration process made use of tissue simulating phantoms as a reference standard with known optical properties similar to those of biological tissue over the NIR range in order to determine $k_{\mathrm{a}}\left(\lambda_{i}\right)$ of sample absorption coefficient using Eq. (2) and to correct for any system response. $k_{\mathrm{a}}\left(\lambda_{i}\right)$ factor is applied later to divide Eq. (2) for future validation of unknown sample optical properties. This calibration procedure was performed immediately before and after each of the experiments in order to monitor system reliability and robustness.

\subsection{Refractive Index Evaluation}

Once the absorption image is recovered [Eq. (2)], a transformation to the imaginary part of the CRI is employed ${ }^{6}$

$k\left(x, y, \lambda_{i}\right)=\frac{\lambda_{i}}{4 \pi} \mu_{\mathrm{a}}\left(x, y, \lambda_{i}\right)$

In this manner, we have a calibrated imaginary CRI cube with dimensions of $x \times y \times 6$, where $x \times y$ is the two-dimensional (2-D) image pixel size and 6 stands for the number of the wavelengths used. By scanning individual columns $\left(x_{\mathrm{i}}\right)$ at each single row $\left(y_{\mathrm{i}}\right)$ of the cube, followed by data fitting using the power-law equation ${ }^{17}$

$k\left(x_{i}, y_{i}, \lambda_{i}\right)=a \lambda_{i}^{1-b}$,

a 2-D spatial map of the real RI at different wavelengths is built concomitantly using the computation in Eq. (1). By averaging each of the obtained $n\left(\lambda_{\mathrm{i}}\right)$ maps, the mean and standard deviation values are derived. Then, the spectral dependence of $n$ can be fitted to the data using one of the following dispersion schemes: ${ }^{18,19}$

Cornu: $n(\lambda)=A+B \cdot(\lambda-C)^{-1}$

Cauchy: $n(\lambda)=A+B \cdot \lambda^{-2}+C \cdot \lambda^{-4}$

Conrady: $n(\lambda)=A+B \cdot \lambda^{-1}+C \cdot \lambda^{-3.5}$

Sellmeier: $n(\lambda)=\sqrt{1+\sum_{i} \frac{B_{i} \cdot \lambda^{2}}{\lambda^{2}-C_{i}}}$,

where $\lambda$ is the wavelength in nanometer. The above fitting coefficients of each equation $(A, B, C, a$, and $b)$ are determined using the least-squares principle.

\subsection{Three-Dimensional Refractive Index Profilometry}

The configuration of our system (Fig. 1) is similar to that used in profilometry-based fringe projection (FPP). ${ }^{21}$ Briefly, when a sinusoidal pattern is projected onto the target object surface, the resultant phase distortions of deformed patterns are quantitatively related to the object's surface height distribution. The intensity of the distorted image recorded by a camera for a single frame is given by

$I_{n}=A(x, y)+B(x, y) \cos [\phi(x, y)+2 \pi n / N] \quad N \geq 3$,

where $I_{n}$ is the $n$-frame sampling intensity, $A(x, y)$ represents the background intensity, $B(x, y)$ is the amplitude of the fringe pattern, and $\phi(x, y)$ is the phase modulation at the measured height. The phase $\phi(x, y)$ can be calculated by traditional phase stepping with $N$ images. For a case of an $N=3$ with a 120-deg phase-shifting step among adjacent patterns, it can be shown that ${ }^{25}$

$\phi(x, y)=\arctan \left[\sqrt{3} \frac{I_{1}(x, y)-I_{3}(x, y)}{2 I_{2}(x, y)-I_{1}(x, y)-I_{3}(x, y)}\right]$,

where $I_{1}\left(\lambda_{i}\right), I_{2}\left(\lambda_{i}\right)$, and $I_{3}\left(\lambda_{i}\right)$ represent the captured adjacent reflectance images at spatial phases of $0 \mathrm{deg}, 120 \mathrm{deg}$, and $240 \mathrm{deg}$, respectively. This phase-shift method has been considered as an essential technique to extract the phase information [Eq. (7)] and employs only simple arithmetic operations. The phase map $\phi(x, y)$ calculated by Eq. (7) is wrapped into the values from $-\pi$ to $\pi$ with discontinuities along $2 \pi$ phase jumps. To remove the phase jumps and to obtain continuous phase distribution, an unwrapping algorithm is carried. For unwrappimg process and to improve sample visualization, we use the phase-derivative-variance (PDV ${ }^{26}$ map following 2-D finite impulse response filter by the Blackman window in MATLAB ${ }^{\mathrm{TM}}$. Once a correct phase unwrapping is achieved, the height can then be computed based upon the system geometry. The relation between phase and object height is written as ${ }^{27}$

$h(x, y)=\frac{(L / d)}{2 \pi f_{x}} \phi(x, y)=K \phi(x, y)$,

where $L$ is the distance between the camera and the object, $d$ is distance between the projector and the camera, and $f_{x}$ is the spatial frequency of the projected fringes. In optical metrology area, Eq. (8) is known as the phase-to-height triangulation conversion algorithm. Once the object profile is obtained, simple visualization of the object's RI can be obtained in 3-D by displaying the image profile as texture map using the following commands in MATLAB ${ }^{\mathrm{TM}}$ software:

$X=2 \mathrm{D}$ matrix of $n$ at single wavelength;

$Y=2 \mathrm{D}$ matrix of the correct phase unwrapping;

figure; $\operatorname{surf}(Y, \quad X$, 'FaceLighting', 'gouraud' ,FaceColor',

'interp', 'AmbientStrength',0.5)

colormap(jet)

shading interp

set(gca, 'fontweight', 'bold' ,'fontsize',14); axis off;

colorbar('horiz'); caxis([1.42, 1.43]);

title('3-D Refractive index', 'fontsize',12, 'fontweight', 'bold');

xlabel(' $\mathrm{X}$ ', 'fontsize',14, 'fontweight', 'bold')

ylabel('Y', 'fontsize',14, 'fontweight', 'bold')

zlabel('Height', 'fontsize', 14, 'fontweight', 'bold')

$\operatorname{view}(24,22)$;

Figure 2 is a flowchart illustrating the above approach to formulate RI and its 3-D representation.

\subsection{Animal Preparation}

The animal experiments used in this study were performed in accordance with ethics protocols approved by the Animal Care and Use Committee at Ariel University, Ariel, Israel. 


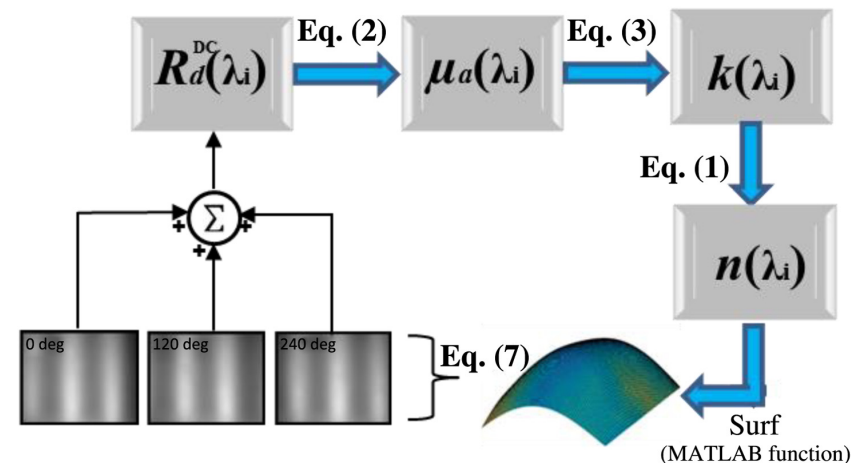

Fig. 2 Flow chart of the process for estimating the RI and its 3-D profile.
Male C57BL/6 mice ( $n=3,27$ to $30 \mathrm{~g}, \sim 12$ weeks old) were housed in the animal facility at the Ariel University with free access to standard laboratory chow and water. Anesthesia was induced by an intraperitoneal injection of ketamine $(80 \mathrm{mg} / \mathrm{kg})$ and xylazine $(20 \mathrm{mg} / \mathrm{kg})$ in sterile saline. The depth of anesthesia was sufficient to eliminate withdrawal from foot pinch, corneal reflex, or vibrissal movements. After the mouse was anesthetized, it was constrained on an in-housemade experimental platform, scalp hair was carefully removed using a commercial hair-removing lotion, and a sponge was inserted underneath the chin. A heating plate precisely controlled by a home-built thermal regulation system was placed under the mouse to control the body temperature, and a digital thermometer was inserted rectally to measure core body temperature. Changes in body temperature were induced by increasing

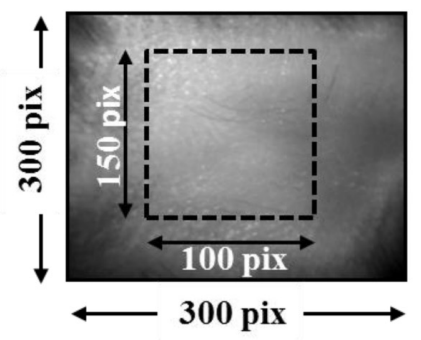

(a)
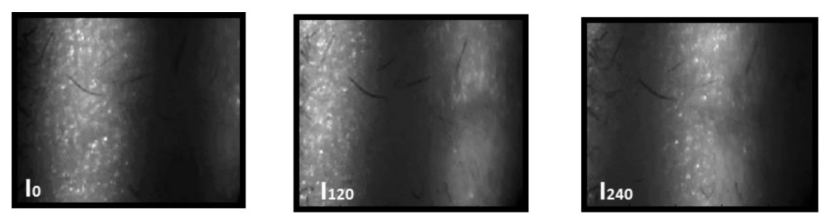

(b)
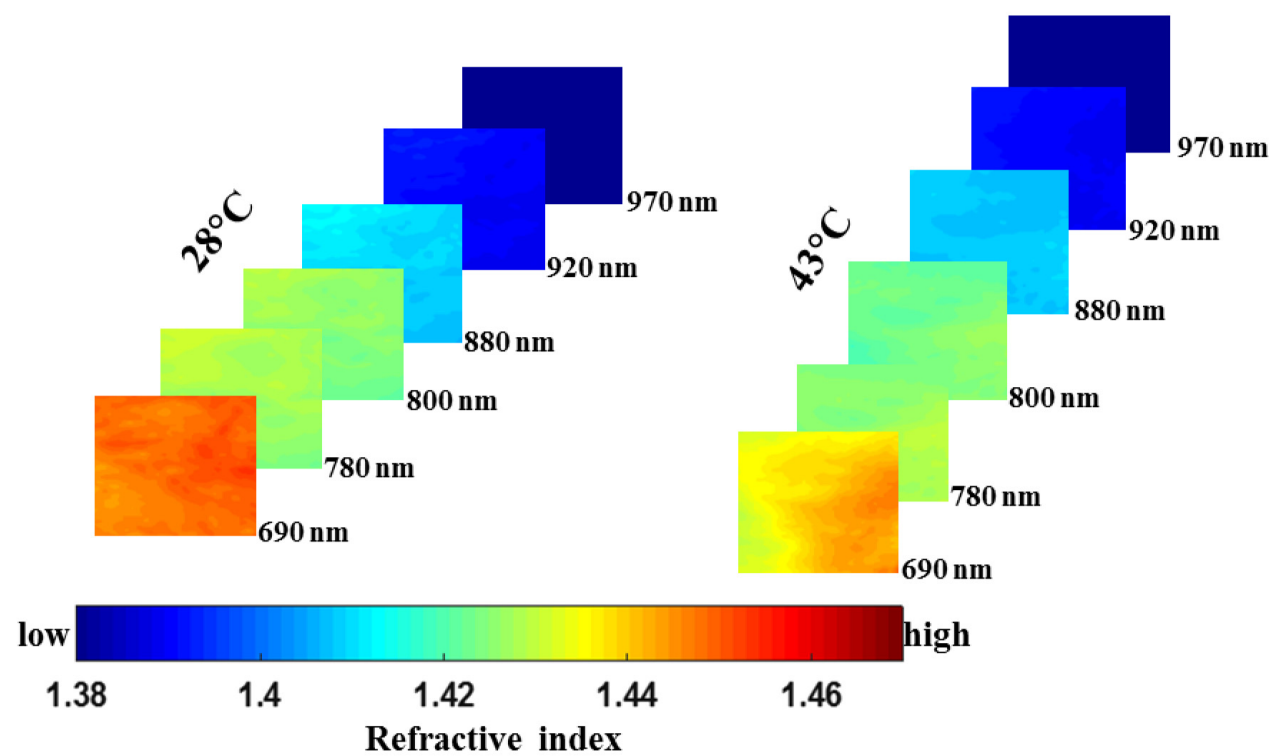

(c)

Fig. 3 (a) Image of the shaved mouse scalp observed by the camera at $690 \mathrm{~nm}$. Dimensions in pixels/ $\mathrm{mm}$ are presented. The broken frame is the ROI selected for analysis. ROI is selected between the anterior coronal suture (bregma) and posterior coronal suture (lambda). (b) Examples of projected structured light patterns at $f_{x}=0.27 \mathrm{~mm}^{-1}$ with phase shift of $120 \mathrm{deg}$ among patterns. (c) Series of 2-D false-color spatial maps of the RI at different wavelengths (hyperspectral data) for two extreme temperatures of $28^{\circ} \mathrm{C}$ and $43^{\circ} \mathrm{C}$. The color bar at the bottom indicates the RI value of each pixel in the map. As demonstrated, change in temperature leads to localized changes in RI within the brain surface. 
the temperature of a folded heating plate. In addition, a thermal camera (FLIR i7 IR, resolution $140 \times 140$ pixels) was positioned slightly off angle from the mouse head to measure brain temperature. Temperature of brain and body was recorded every 2 min during which the mouse body core temperature was raised from $28^{\circ} \mathrm{C}$ to $43^{\circ} \mathrm{C}$.

\section{Results and Discussion}

Figure 3(a) is a typical planner illumination reflectance image of a representative mouse scalp captured at $690 \mathrm{~nm}$, alongside the
ROI (dashed rectangle) situated between the anterior coronal suture (bregma) and posterior coronal suture (lambda). Three representative modulated images captured by the camera at a single spatial frequency of $f_{x}=0.27 \mathrm{~mm}^{-1}$, at respective phase shifts of $0 \mathrm{deg}, 120 \mathrm{deg}$, and $240 \mathrm{deg}$, are shown in Fig. 3(b). Following the processing algorithm described in Sec. 2.4, a series of 2-D false-color spatial maps of the RI at different wavelengths (hyperspectral data) for two extreme temperatures of $28^{\circ} \mathrm{C}$ and $43^{\circ} \mathrm{C}$ are shown in Fig. 3(c). The color bar at the bottom indicates the RI value of each pixel in the map.

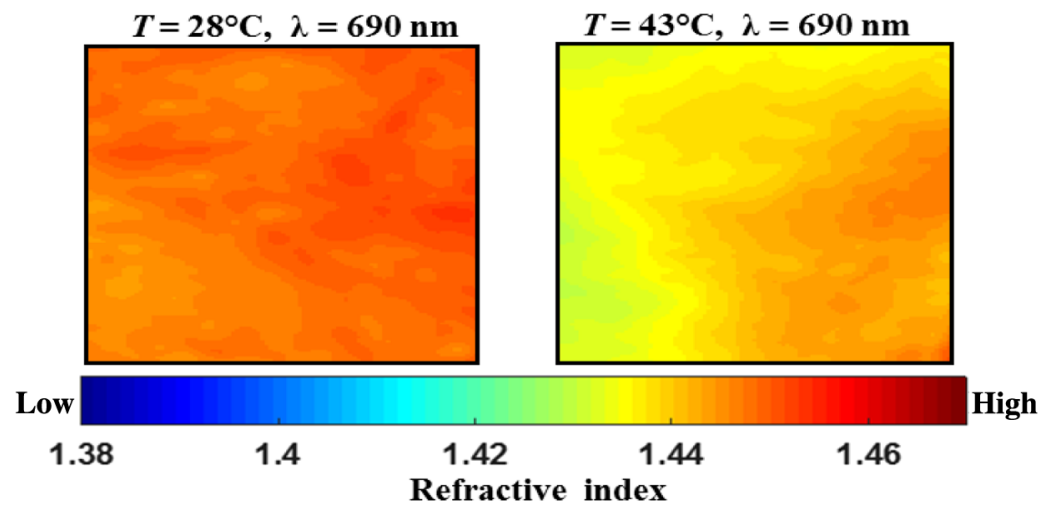

(a)
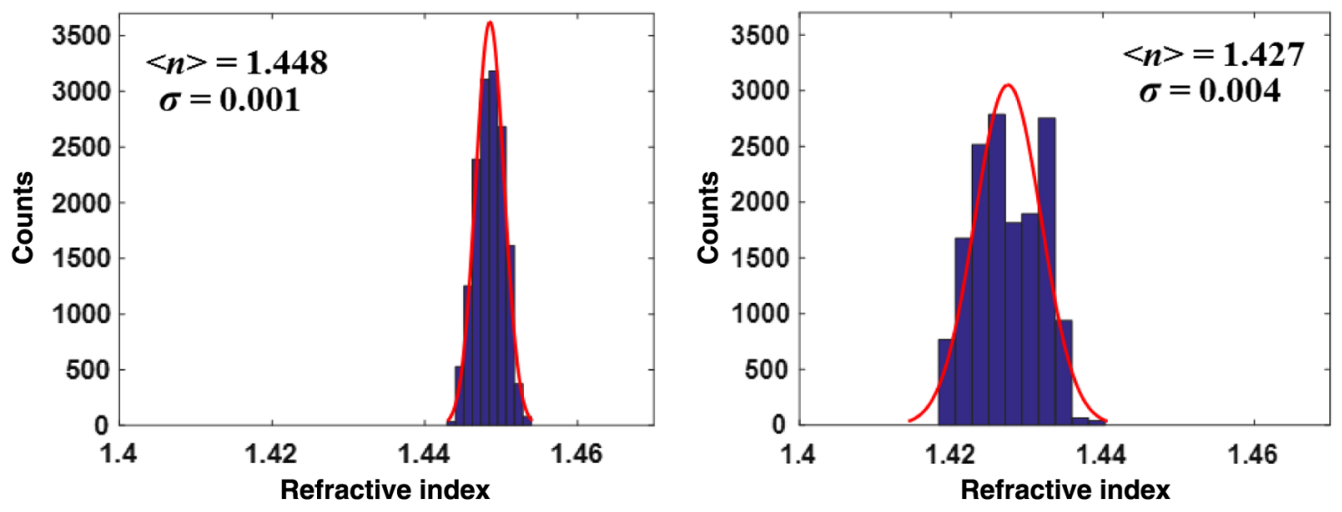

(b)

Fig. 4 (a) The first two maps at $690 \mathrm{~nm}$ from the spectral cube in Fig. 3(c) and their corresponding pixel histogram profiles (b) for the two extreme temperatures. The color scale below the maps shows the value of each pixel; higher index values correspond to brighter pixels and vice versa. The vertical axis in the histogram reflects the number of counts in each bin, while the solid curve is a Gaussian fit with appropriate mean and standard deviation. Conversion of each map into histogram distributions demonstrated localized changes in minimum, maximum, and average RI values at single-pixel resolution, revealing spatial heterogeneity of RI values within the FOV.

Table 1 Coefficients of the four dispersion equations [Eq. (5), in this paper] at $28^{\circ} \mathrm{C}$ and $43^{\circ} \mathrm{C}$. Coefficients were obtained with wavelength in the unit of nanometers.

$28^{\circ} \mathrm{C}$

\begin{tabular}{lll}
\hline Cauchy & $A=1.4057, B=9.686, C=-0.4$ & $A=1.4047, B=10.0145, C=0.1$ \\
Sellemier & $B 1=345.3624, C 1=393.5024$ & $B 1=335.0951, C 1=380.2549$ \\
& $B 2=-344.73, C 2=-283.6719$ & $B 2=-334.4449, C 2=-275.8240$ \\
Cornu & $A=1.4032, B=1.3007, C=-28.7977$ & $A=1.4032, B=1.3007, C=-28.7978$ \\
Conrady & $A=1.4057, B=11.1979, C=1.2$ & $A=1.4047, B=11.1979, C=1.2$ \\
\hline
\end{tabular}


As depicted, change in temperature leads to localized changes in RI within the brain tissue morphology. These RI alterations have been shown to represent gross morphological changes in the mean size, density, and distribution of cell membrane, nuclei, lysosomes, peroxisomes, protein, DNA, mitochondria, and other organelles, as well as hemodynamic changes in hemoglobin concentration, water, lipids, and oxygen saturation levels. The first two color-coded maps at $690 \mathrm{~nm}$ from the spectral cube in Fig. 3(c) and their corresponding pixel histogram profiles for the two temperatures are shown in Figs. 4(a) and 4(b), respectively. The color scale below shows the value of each pixel in the map, such that higher index values correspond to brighter pixels and overall reveal spatial heterogeneities within the measured area. The vertical axis in the histogram reflects the number of counts in each bin while the solid curve is a Gaussian fit with appropriate mean and standard deviation. Conversion of each

Table 2 Coefficients of the four dispersion equations at $28^{\circ} \mathrm{C}$ and $43^{\circ} \mathrm{C}$ following second derivative. Coefficients were obtained with wavelength in nanometers.

\begin{tabular}{llc}
\hline & \multicolumn{1}{c}{$28^{\circ} \mathrm{C}$} & \\
\hline Cauchy & $A=0.0012, B=10, C=0.0001$ & $A 3^{\circ} \mathrm{C}$ \\
Sellemier & $B 1=-0.5, C 1=-0.0001$ & $B 1=-0.499, C 1=-0.0003$ \\
& $B 2=-0.5, C 2=-0.0001$ & $B 2=-0.5001, C 2=-0.0004$ \\
Cornu & $A=-0.018, B=2.4991, C=0.0001$ & $A=-0.0014, B=2.0053, C=0.0001$ \\
Conrady & $A=-0.0018, B=2.4991, C=0.0001$ & $A=-0.0014, B=2.0053, C=0.001$ \\
\hline
\end{tabular}

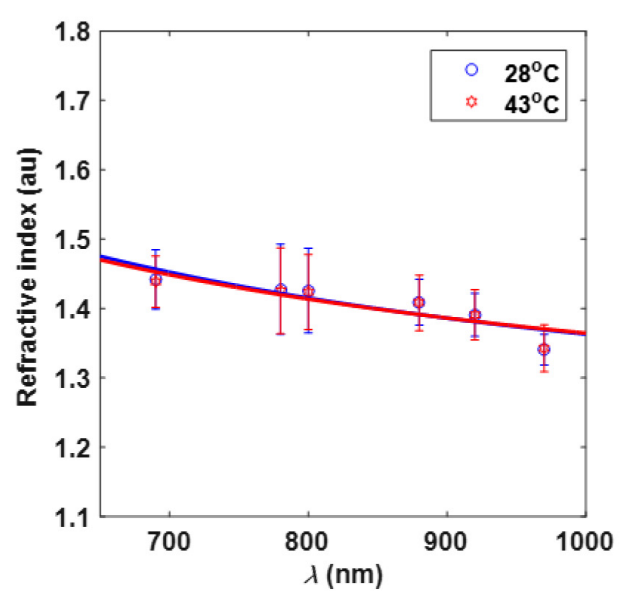

(a)

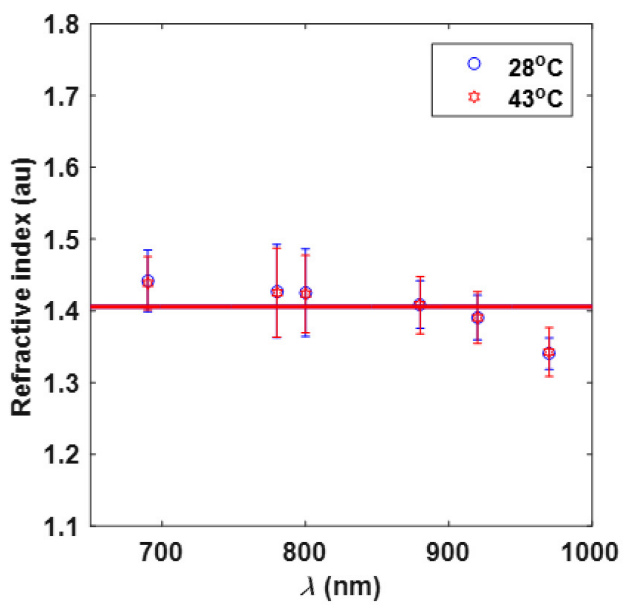

(c)

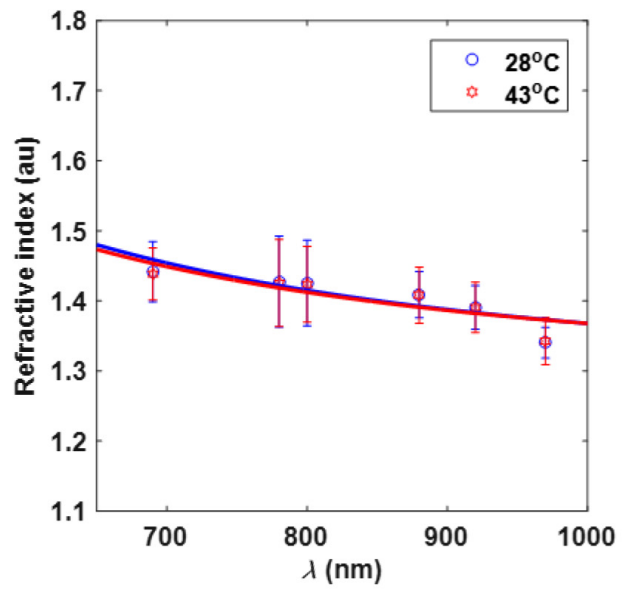

(b)

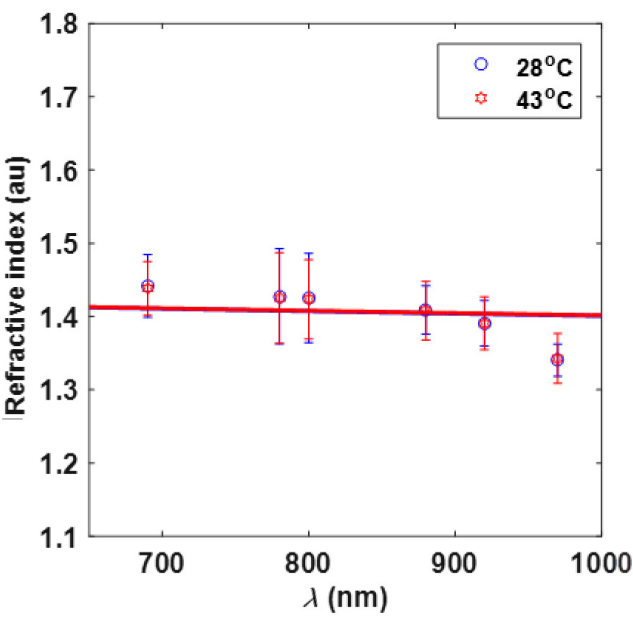

(d)

Fig. $5 \mathrm{RI}$ spectra at two extreme temperatures of $28^{\circ} \mathrm{C}$ and $43^{\circ} \mathrm{C}$ for (a) Conrady, (b) Sellemier, (c) Cauchy, and (d) Cornu models. Data points represent the mean and a bar refers to standard error (correspond to the variation between ROI pixels). The line represents the model fitting to experimental data. 
map into histogram distributions demonstrated localized changes in minimum, maximum, and average RI values at single-pixel resolution, revealing spatial heterogeneity of RI values within the FOV. The RI variations with increased temperature clearly demonstrated by both $x$ - and $y$-axes of the histograms indicate morphological and hemodynamic variations and overall brain dysfunction during thermal insult. ${ }^{28-30}$

By averaging the RI maps of each wavelength in Fig. 3(c), a single mean and standard deviation correspond to the variation of RI values of the ROI pixel is obtained. Based upon this information, RI spectra were then derived by the wavelength-dependent dispersion model [Eq. (5)]. The wavelength dependence of RI for the two extreme temperatures is plotted in Fig. 5, according to the four models described in Eq. 5, Sec. 2.4. Data points represent the discrete calculated mean RI and its standard deviation at specific wavelengths. The lines are fitted based on dispersion models to show the spectral dependence of RI values. The fitting coefficients of each dispersion model $(A$, $B$, and $C$ ) are shown in Table 1 . In contrast to Cauchy [Fig. 5(c)] and Cornu [Fig. 5(d)] models, which present a nearly constant RI value of $\sim 1.4$ across the wavelengths, both Conrady [Fig. 5(a)] and Sellemier [Fig. 5(b)] models yield exponential monotone spectra in which the RI slightly decreases by $\sim 8 \%$ $\left(28^{\circ} \mathrm{C}: 1.475 \rightarrow 1.363,43^{\circ} \mathrm{C}: 1.48 \rightarrow 1.368\right)$. Thus, a moderate reduction in RI from 690 to $970 \mathrm{~nm}$ was observed as the wavelength increased. The plots obtained using Cauchy and Cornu equations do not stand well with previous publications demonstrating decreased RI as the wavelength increases. ${ }^{2,17,18,31,32}$ As seen, along the spectrum of Figs. 5(a) and 5(b), there are minute differences between RI levels with changes in temperature. The lack of substantial alterations in RI levels between temperatures may have several origins, such as: (i) approximations made using Eqs. (1)-(4), (ii) utilizing a limited number of NIR wavelengths, (iii) the partial volume effect, ${ }^{11,33,34}$ and (iv) errors related to spatial frequency domain. ${ }^{35,36}$ Nevertheless, to increase discrepancies in RI spectra with temperature, a second-derivative operation was implemented on each image map appearing in Fig. 3(c). The "imgradient" function in MATLAB $^{\mathrm{TM}}$ with prewitt method (arbitrarily chosen) was used twice for this purpose. Following second derivation, a single mean value was obtained by averaging the derivative image for each wavelength, and the wavelength dependence of RI was subsequently obtained using one of the dispersion equations. The corresponding fitting coefficient of each model for the two given temperatures is listed in Table 2, and the plotted derivative spectrum for the Conrady model is shown in Fig. 6(a). In contrast to the Cauchy model, which yields a constant value across the wavelengths with $16 \%$ contrast among temperatures Fig. 6(b), or the Sellemier scheme, which revealed opposite behavior in temperatures (not appear), the Conrady model shows a constant spectral decrease from 690 to $970 \mathrm{~nm}$, while the RI spectrum lowers with a temperature increase resulting in distinguishable differences among temperatures. For example, an $18 \%$ difference is observed at $690 \mathrm{~nm}$, with a $14 \%$ difference at $970 \mathrm{~nm}$. Following data analysis, we found Cornu wavelength-dependent fitting to yield similar results to those of Conrady shown in Fig. 6(a). Based on Figs. 5 and 6, we found fitting data with Conrady method to be more reasonable than the other three schemes, supporting the use of second-derivative operation to highlight variations in RI arising with temperature. In Ref. 10, Fig. 9 shows changes in RI of biological tissue occurring with altered tissue density, as measured by Abbe

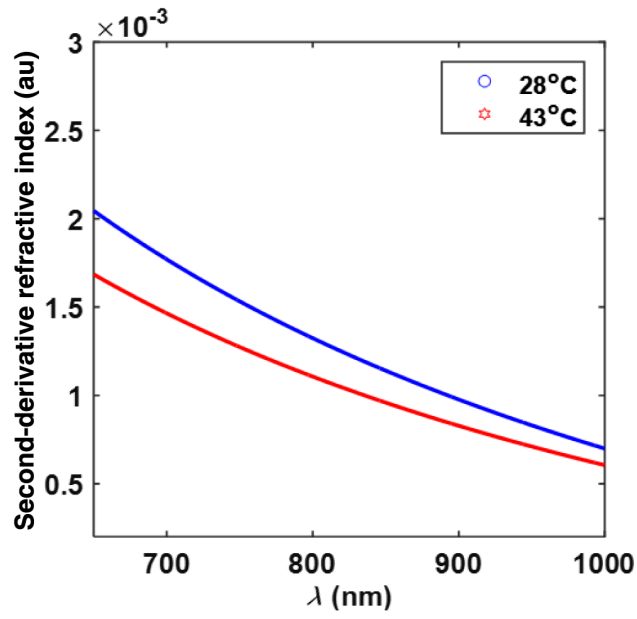

(a)

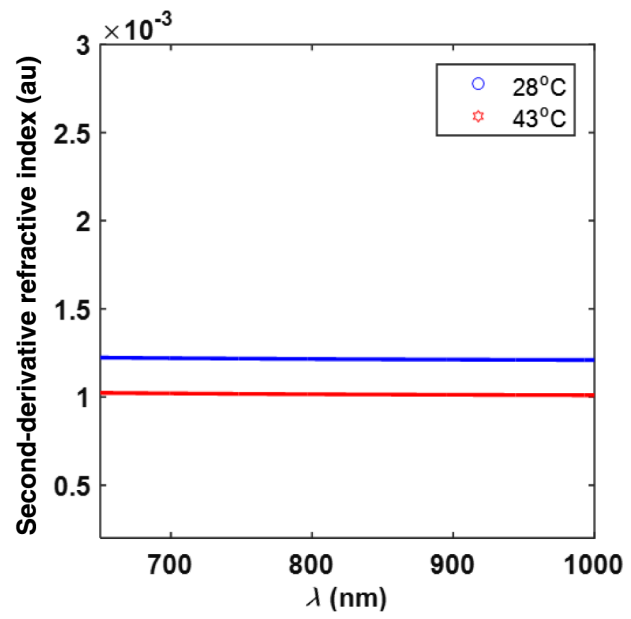

(b)

Fig. 6 Second-derivative RI for: (a) Conrady and Cornu, and (b) Cauchy. Second-derivative operation was used to highlight variations in Rl occurring with temperature.

refractometer. Similarly, our previous studies demonstrated structural variations occurring during heatstroke through altered reduced scattering coefficient, which linked to the changes in RI. ${ }^{37}$ Thus, we may argue temperature change causes changes in tissue density, which in turn alters tissue RI. On the other hand, this argumentation can be supported by the GladstoneDale (G-D) equation, which makes the connection between material density $(\rho)$ and its RI $(n)$ following: $n=1+k \rho$, where $k$ is the $\mathrm{G}-\mathrm{D}$ constant. ${ }^{38}$ The overall changes in RI over a continuous spectral NIR region highlight the pathophysiologic changes occurring in the brain in response to thermal insult and demonstrate our ability to resolve an additional intrinsic optical parameter.

In the final step, RI distribution is presented in 3-D [Figs. 7(a) and 7(b)] showing the head RI at both temperature extremes at a single wavelength $(690 \mathrm{~nm})$ generated according to the FPP principle (Sec. 2.5). For distinguishable visualization, the color bar representing the RI is slightly different across the head surface. The dashed lines highlight the changes in the surface occurring with temperature elevation. The use of unwrapping methodology and spatial filtering provided clear topology of the mouse head. Thermal images demonstrating temperature distribution across the mouse body acquired by a thermographic 


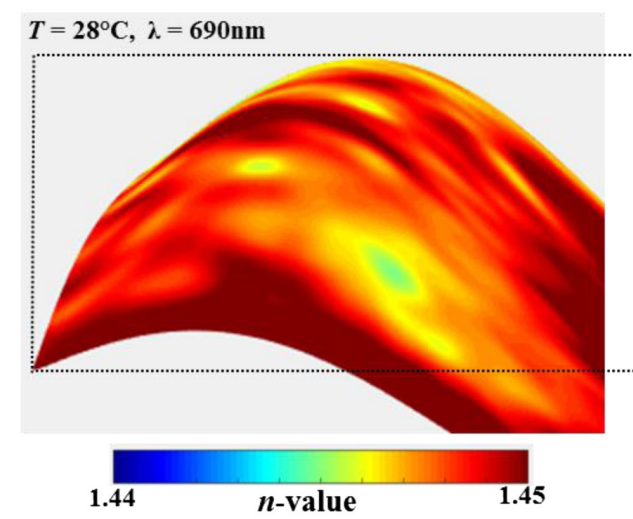

(a)

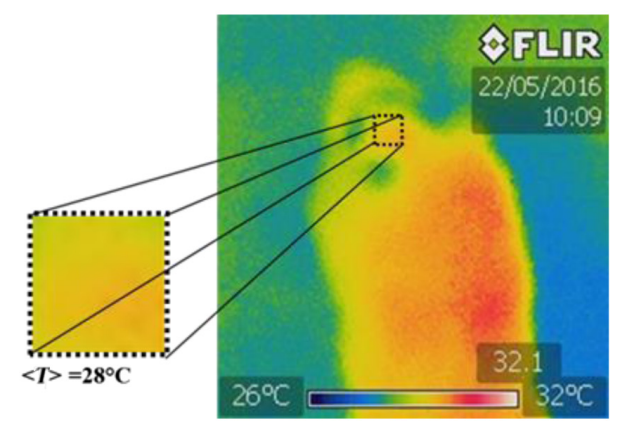

$T=43^{\circ} \mathrm{C}, \lambda=690 \mathrm{~nm}$

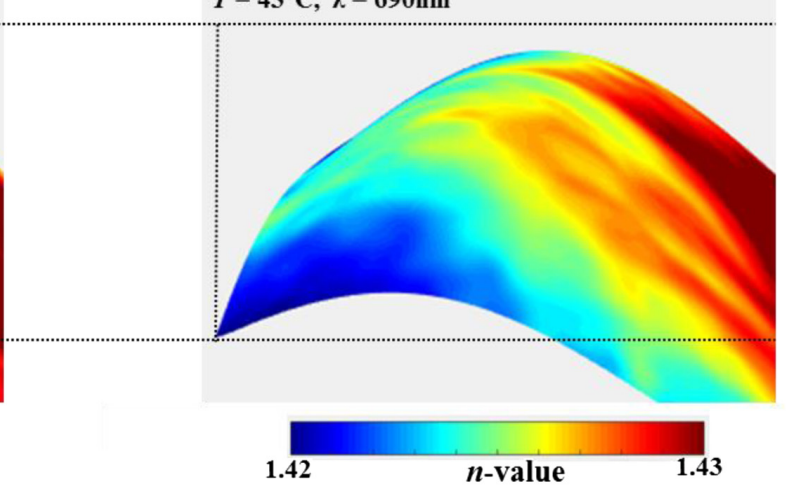

(b)

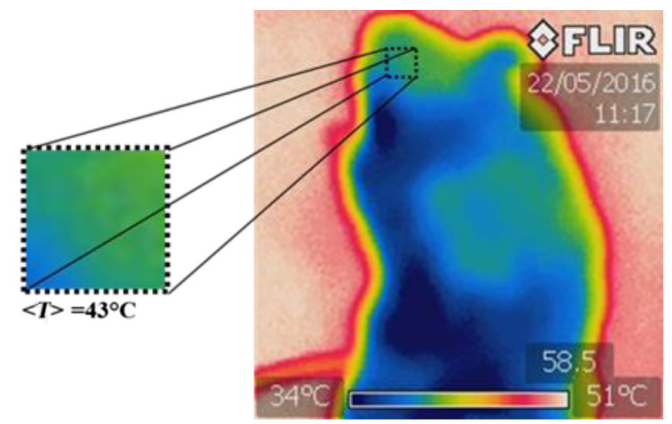

(c)

Fig. 7 Three-dimensional head RI distribution at (a) $28^{\circ} \mathrm{C}$ and (b) $43^{\circ} \mathrm{C}$ at $690 \mathrm{~nm}$. The color bar representing the $\mathrm{RI}$ values across the head surface. The dashed lines highlight the changes in the topology occurring with temperature elevation. (c) Thermal images were captured by thermal camera demonstrating temperature distribution across the mouse body and head.

camera [Fig. 7(c)] clearly demonstrate differences in head temperature level.

\section{Concluding Remarks}

In this work, spatially modulated NIR imaging, KK processing, and dispersion models were used in tandem to estimate the real RI of a mouse brain following thermal insult. As expected, change in temperature lead to alterations in tissue RI, which reflect changes in brain tissue morphology and hemodynamics. Furthermore, the wavelength dependence of the RI was demonstrated using four well-known dispersion schemes: Cornu, Cauchy, Conrady, and Sellmeier. A decrease of $\sim 8 \%$ in RI across the NIR spectrum was seen and second-derivative procedure further enhanced differences among temperatures. Results found the Conrady formula to be more accurately than the other tested dispersion schemes. In a wider context, quantification of the RI can contribute to: (1) assessment of physiologic changes, (2) treatment response, and (3) modeling light propagation in scattering media. Additionally, 3-D spatial distribution of RI values as a function of temperature was demonstrated based on FPP principle. PDV and spatial filtering were then used for unwrapping, providing a smooth surface topology of RI distribution in the mouse brain. This mapping technique suggests an additional capability of the spatially modulated NIR imaging system to quantitatively characterize RI values in 3-D. Since fringe projection is the basis of both spatially MI and object surface profilometry, it enables us to simultaneously estimate RI values and to profile RI distribution in 3-D independent of additional hardware or software. ${ }^{23}$ This simultaneity is a unique strength of the spatially modulated system in comparison to others. For the reader information, 3-D RI distribution at subdiffraction resolutions using structured light in microscopy setup and diffraction tomography computation was documented recently. ${ }^{39}$ Overall, this work offers an easier, simpler, and lower-cost framework for retrieving CRI components of biological tissue in contrast to others. ${ }^{3,40,41}$

\section{Disclosures}

The authors have no relevant financial interests in this article and no potential conflicts of interest to disclose.

\section{References}

1. J.-C. Lai et al., "Complex refractive index measurement of biological tissues by attenuated total reflection ellipsometry," Appl. Opt. 49(16), 3235-3238 (2010).

2. P. Giannios et al., "Complex refractive index of normal and malignant human colorectal tissue in the visible and near-infrared," $J$. Biophotonics 10(2), 303-310 (2017).

3. T.-Q. Sun et al., "Measurement of complex refractive index of turbid media by scanning focused refractive index," Opt. Lett. 41(16), 3767-3770 (2016).

4. Q. Ye et al., "Measurement of the complex refractive index of tissuemimicking phantoms and biotissue by extended differential total reflection method," J. Biomed. Opt. 16(9), 097001 (2011).

5. J. S. Maier et al., "Possible correlation between blood glucose concentration and the reduced scattering coefficient of tissues in the near infrared," Opt. Lett. 19(24), 2062-2064 (1994).

6. J. Gienger et al., "Determining the refractive index of human hemoglobin solutions by Kramers-Kronig relations with an improved absorption model," Appl. Opt. 55(31), 8951-8961 (2016). 
7. H. Liu et al., "Real part of refractive index measurement approach for absorbing liquid," Appl. Opt. 54(19), 6046-6052 (2015).

8. J. Wang et al., "Measurement of the refractive index of hemoglobin solutions for a continuous spectral region," Biomed. Opt. Express 6(7), 2536-2541 (2015).

9. J. Räty, P. Pääkkönen, and K-E. Peiponen, "Assessment of wavelength dependent complex refractive index of strongly light absorbing liquids," Opt. Express 20(3), 2835-2843 (2012).

10. S. L. Jacques, "Optical properties of biological tissues: a review," Phys. Med. Biol. 58(11), R37-R61 (2013).

11. D. J. Cuccia et al., "Quantitation and mapping of tissue optical properties using modulated imaging," J. Biomed. Opt. 14(2), 024012 (2009).

12. S. Gioux et al., "Three-dimensional surface profile intensity correction for spatially modulated imaging," J. Biomed. Opt. 14(3), 034045 (2009).

13. T. D. O'Sullivan et al., "Diffuse optical imaging using spatially and temporally modulated light," J. Biomed. Opt. 17(7), 071311 (2012).

14. S. C. Kanick et al., "Sub-diffusive scattering parameter maps recovered using wide-field high-frequency structured light imaging," Biomed. Opt. Express 5(10), 3376-3390 (2014).

15. M. Sharma et al., "Verification of a two-layer inverse Monte Carlo absorption model using multiple source-detector separation diffuse reflectance spectroscopy," Biomed. Opt. Express 5(1), 40-53 (2014).

16. G. Zonios and A. Dimou, "Light scattering spectroscopy of human skin in vivo," Opt. Express 17(3), 1256-1267 (2009).

17. P. Giannios et al., "Visible to near-infrared refractive properties of freshly-excised human-liver tissues: marking hepatic malignancies," Sci. Rep. 6, 27910 (2016).

18. H. Ding et al., "Refractive indices of human skin tissues at eight wavelengths and estimated dispersion relations between 300 and $1600 \mathrm{~nm}$," Phys. Med. Biol. 51, 1479-1489 (2006)

19. B. Tatian, "Fitting refractive-index data with the Sellmeier dispersion formula," Appl. Opt. 23(4), 4477-4485 (1984).

20. V. Srinivasan, H. C. Liu, and M. Halioua, "Automated phase-measuring profilometry of 3-D diffuse objects," Appl. Opt. 23(18), 3105-3108 (1984).

21. S. S. Gorthi and P. Rastogi, "Fringe projection techniques: whither we are?" Opt. Lasers Eng. 48(2), 133-140 (2010).

22. S. V.-D. Jeught and J. J. J. Dirckx, "Real-time structured light profilometry: a review," Opt. Lasers Eng. 87, 18-31 (2016).

23. M. van de Giessen, J. P. Angelo, and S. Gioux, "Real-time, profilecorrected single snapshot imaging of optical properties," Biomed. Opt. Express 6(10), 4051-4062 (2015).

24. X. He et al., "Nondestructive determination of optical properties of a pear using spatial frequency domain imaging combined with phase-measuring profilometry," Appl. Opt. 56(29), 8207-8214 (2017).
25. P. S. Huang and S. Zhang, "Fast three-step phase-shifting algorithm," Appl. Opt. 45(21), 5086-5091 (2006).

26. Y. Zhang et al., "An improved quality guided phase unwrapping method and its applications to MRI," Prog. Electromagn. Res. 145, 273-286 (2014).

27. H. Fan, H. Zhao, and Y. Tan, "Automated three-dimensional surface profilometry using dual-frequency optic fiber phase-shifting method," Opt. Eng. 36(11), 3167-3171 (1997).

28. H. Wang et al., "Brain temperature and its fundamental properties: a review for clinical neuroscientists," Front. Neurosci. 8, 307 (2014).

29. A. Bouchama and J. P. Knochel, "Heat stroke," N. Engl. J. Med. 346(25), 1978-1988 (2002).

30. E. J. Walter and M. Carraretto, "The neurological and cognitive consequences of hyperthermia," Crit. Care 20, 199 (2016).

31. T. Vo-Dinh, Biomedical Photonics Handbook, Second Edition: Fundamentals, Devices, and Techniques, CRC Press, Boca Raton, Florida (2015)

32. D. G. Stavenga, H. L. Leertouwer, and B. D. Wilts, "Quantifying the refractive index dispersion of a pigmented biological tissue using Jamin-Lebedeff interference microscopy," Light Sci. Appl. 2, e100 (2013).

33. S. D. Konecky et al., "Quantitative optical tomography of sub-surface heterogeneities using spatially modulated structured light," Opt. Express 17(17), 14780-14790 (2009).

34. M. A. G. Ballester, A. P. Zisserman, and M. Brady, "Estimation of the partial volume effect in MRI,” Med. Image Anal. 6(4), 389-405 (2002).

35. N. Bodenschatz et al., "Sources of errors in spatial frequency domain imaging of scattering media," J. Biomed. Opt. 19(7), 071405 (2014).

36. Y. Zhao et al., "Angle correction for small animal tumor imaging with spatial frequency domain imaging (SFDI)," Biomed. Opt. Express 7(6), 2373-2384 (2016).

37. D. Abookasis et al., "Diffuse near-infrared reflectance spectroscopy during heatstroke in a mouse model: pilot study," J. Biomed. Opt. 17(10), 105009 (2012).

38. C. Yun-Yun et al., "Extension of the Gladstone-Dale equation for flame flow field diagnosis by optical computerized tomography," Appl. Opt. 48(13), 2485-2490 (2009).

39. S. Chowdhury et al., "Refractive index tomography with structured illumination," Optica 4(5), 537-545 (2017).

40. W. R. Calhoun et al., "Measurement of the refractive index of highly turbid media," Opt. Lett. 35(8), 1224-1226 (2010).

41. M. L. Dong et al., "Accurate in situ measurement of complex refractive index and particle size in intralipid emulsions," J. Biomed. Opt. 18(8), 087003 (2013).

Biographies for the authors are not available. 